\title{
Traumatic Floating Clavicle: A Case Report
}

\author{
Choo CY, MD (USM), HY Wong, MS Orth (UKM), Nordin A, MMed Orth (USM)
}

Department of Orthopaedics and Traumatology, Hospital Seberang Jaya, Seberang Jaya, Malaysia

\begin{abstract}
Shoulder girdle injuries after high energy traumatic impacts to the shoulder have been well documented. Based on the series of 1603 injuries of the shoulder girdle reported by Cave and colleagues, $85 \%$ of the dislocations were glenohumeral, $12 \%$ acromioclavicular and $3 \%$ sternoclavicular ${ }^{1}$. Less frequently described are injuries involving both the sternoclavicular and acromioclavicular joints simultaneously in one extremity. The present report discusses a case of traumatic floating clavicle associated with ipsilateral forearm and wrist injury which was treated surgically.
\end{abstract}

Key Words:

Floating clavicle, bipolar dislocation, sternoclavicular

\section{INTRODUCTION}

Dislocation of both ends of the clavicle, also known as bipolar clavicular dislocation, traumatic floating clavicle or panclavicular dislocation is a rare injury, with approximately 40 cases reported in published literature ${ }^{1}$. Generally it is related to major trauma such as high-speed road traffic accident, fall from height or heavy object falling on to the shoulder, although it can also occur with lesser trauma. It was first described by Porral in 1831, then, Beckman reported 16 cases in $1924^{3}$ and the 17 th case was added by Gearen and Petty in $1982^{4}$. Sanders and associates treated 6 patients in $1990^{5}$, and other authors have published isolated cases. Here, we present a case of successful open reduction and internal fixation of this injury. We would like to highlight a new method of surgical treatment using Mersilene tape (synthetic material) for reconstruction. Since this diagnosis is easily missed, we emphasize that high index of suspicion is required for diagnosis.

\section{CASE REPORT}

A 48-year old policeman who was involved in a road traffic accident was diagnosed with cerebral concussion and closed fractures midshaft of the left radius and ulna by a casualty doctor in an outlying hospital. He was referred to a tertiary hospital for further treatment.
On examination, he was clinically stable. Other than previously diagnosed injuries, prominent swelling over the medial end of the left clavicle was noted and there was point tenderness over the left sternoclavicular and acromioclavicular joints. The medial end of the left clavicle was displaced upward and mobile. No tenderness over scapula and equal interscapular distance was noted. Range of motion of the left shoulder was decreased but neurovascular deficit distal to the injury. Other system examinations were unremarkable.

Initial chest radiography revealed superior displacement of the left clavicle with widening of the left sternoclavicular joint, raising suspicion of dislocation of the sternoclavicular joint. Left shoulder radiographs showed marked widening of the acromioclavicular joint space (type V injury - Rockwood classification) and left forearm radiographs revealed comminuted midshaft fracture of the radius, ulnar styloid fracture and distal radioulnar joint dislocation. In light of the high clinical suspicion for sternoclavicular joint injury, a proper anteroposterior chest radiograph with both clavicles clearly outlined was repeated for comparison. This showed displacement of the left clavicle superiorly with increased joint spaces at both ends of left clavicle.

He was treated operatively with sternoclavicular joint reconstruction, hook plating of the left acromioclavicular joint, plating of the left radius and ulna, and K-wiring of the left distal radioulnar joint. The medial clavicle and manubrium were tied under tension in a figure of eight form using Mersilene tape while another length of Mersilene tape was passed around the medial clavicle and the first rib and tied securely under tension. The coracoclavicular ligament was reconstructed with Mersilene tape.

Pendulum exercises, isometric internal and external rotation shoulder strengthening were started 3 days post-operatively. Two weeks later, he was started on biceps and triceps strengthening. The arm was supported with an arm sling for up to 6 weeks. The sling was then discontinued and active range of motion exercises for the shoulder and elbow were started. Overhead activities, light stretching and strengthening exercises progressed gradually. Five months following surgery, his left shoulder had regained full range of movement and full strength of motor power. There was no 


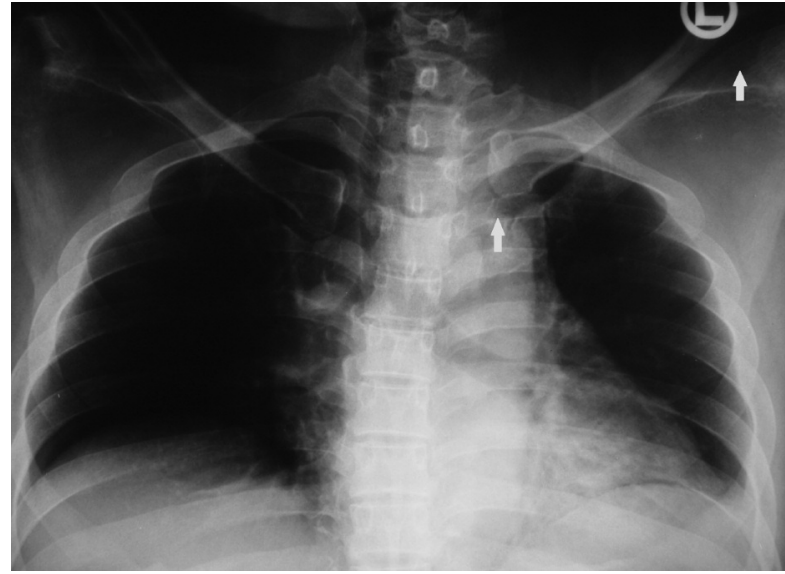

Fig. 1: Chest radiograph showing dislocation of both ends of the clavicle (arrows).

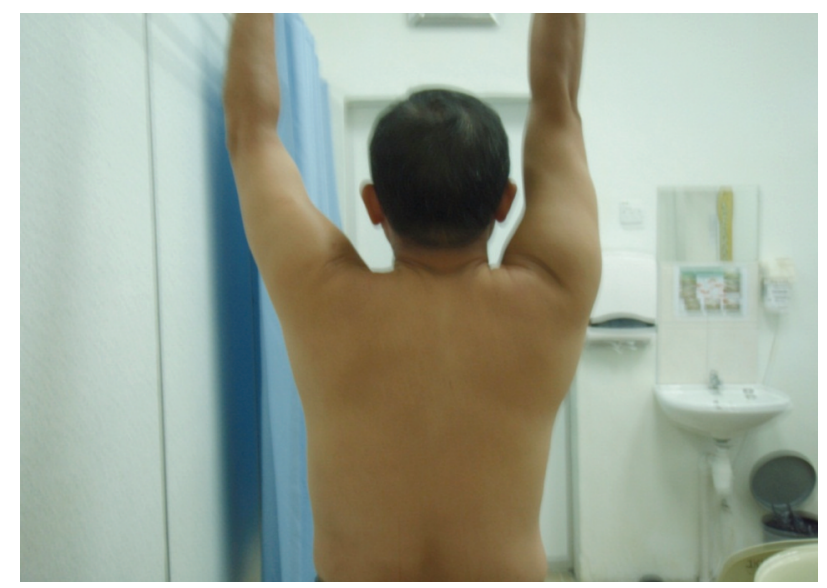

Fig. 3: Full abduction of the shoulders indicating good functional recovery.

prominent or unsightly scarring. He returned to work 4 months post-trauma.

\section{DISCUSSION}

Bipolar dislocation of the clavicle is infrequent, as seen by the scarcity of clinical reports. Due to the rarity of this injury, it is easily missed or delayed in diagnosis especially by junior doctors. It is difficult to detect this injury because the history is often non-specific and physical findings can be subtle. In this case, the diagnosis was initially missed by casualty doctors at an outlying hospital and at our hospital. A high index of suspicion and imaging results aided us in obtaining the diagnosis.

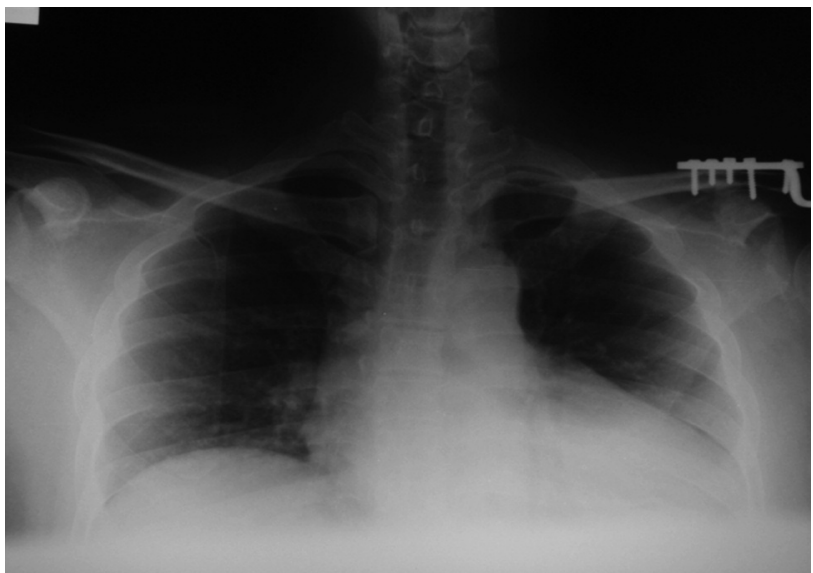

Fig. 2: Left clavicle reduced after operative procedure. The symmetry between the right and the left articular spaces can be observed.

Traumatic floating clavicle is a potentially disabling injury. No standard modality of treatment has been agreed upon. In general, most orthopaedics surgeons recommend a trial of conservative management especially for asymptomatic and low functional demand patients. Although complaints of poor cosmetic results are frequent, patients are typically satisfied with shoulder girdle function. If the patient continues to have symptoms or functional inability despite conservative treatment or when the shoulder cannot be stabilized using closed treatment methods, surgical options must be entertained. Most surgeons agree that the acromioclavicular lesion should be managed as if it was an isolated injury and the sternoclavicular injury should be disregarded even though it may be unstable, because of secondary operative complications.

Our patient was treated operatively because of his age, higher functional demands as a policeman, the knowledge that even after successful closed reduction, residual instability often remains with type $\mathrm{V}$ acromioclavicular joint dislocation and the fact that it is difficult to maintain reduction merely with orthosis.

Use of a hook plate with Mersilene tape for acromioclavicular joint reconstruction results in stable fixation, and reconstruction of the sternoclavicular joint using Mersilene tape is an acceptable surgical technique since the material is readily available and its use offers less morbidity compared to muscle or tendon grafting. Since a good short-term functional outcome was achieved in this patient, this novel method may be useful for reconstruction of the sternoclavicular joint. However, long-term results are still uncertain and further follow-up of this patient is required. 


\section{REFERENCES}

1. Iannotti JP, Williams GR. Disorders of the shoulder: diagnosis \& management; Acromioclavicular and sternoclavicular joints 2007; 2: 979-1050.

2. Porral A. Observation of a double dislocation of the right clavicle (in French). J Univ Hebd Med Chir Prat. 1831; 2: 78-82

3. Beckman T. A case of simultaneous luxation of both ends of the clavicle. Acta Chir Scandinavica. 1924; 56: 156-63.

4. Gearen PF., Petty W. Panclavicular dislocation. Report of a case. J Bone Joint Surg Am. 1982; 64(A): 454-5.

5. Sanders JO, Lyons FA, Rockwood CA Jr. Management of dislocations of both ends of the clavicle. J Bone and Joint Surg Am. 1990; 72: 399-402. 